Molero Lopez-Barajas, D. (2007). Rendimiento académico y opinión sobre la docencia del alumnado participante en experiencias piloto de implantación del Espacio Europeo de Educación Superior.

RELIEVE, v. 13, n. 2, p. 175-190. http://www.uv.es/RELIEVE/v13n2/RELIEVEv13n2_2.htm

\title{
REIIदVE
}

Revista ELectrónica de Investigación

y EValuación Educativa

\section{RENDIMIENTO ACADÉMICO Y OPINIÓN SOBRE LA DOCENCIA DEL ALUMNADO PARTICIPANTE EN EXPERIENCIAS PILOTO DE IMPLANTACIÓN DEL ESPACIO EUROPEO DE EDUCACIÓN SUPERIOR}

\section{[Academic performance and students' teaching assessment in pilot experiments of European Higher Education]}

por

Article record

$\underline{\text { About authors }}$

HTML format $\underline{\text { Ficha del artículo }}$

$\underline{\text { Sobre los autores }}$

Formato HTML

\begin{abstract}
In this work we show a study based on an analysis of academic marks as well as on a teaching valuation of a students sample participating in a pilot experience for the European Credit System Implantation in the Music Teachers Instruction Course. We also detail the way of evaluating the students and the resources used to valuate the teaching. We analyse the differences between the student's marks and their opinion about the teaching in two students groups, one of them using a traditional teaching system and the other one using such experience for the European Credit implantation.
\end{abstract}

\section{Keywords}

Academic Achievement; Student Evaluation of Teacher Performance; European Higher Education Area, Higher Education.

\begin{abstract}
Resumen
En este trabajo presentamos un estudio basado en el análisis de las calificaciones académicas y la valoración de la docencia en una muestra de estudiantes participantes en la experiencia piloto de implantación del crédito europeo en la titulación de Maestro Educación Musical. Detallamos las vías empleadas para evaluar al alumnado y los recursos utilizados para valorar la docencia, analizamos la existencia de diferencias entre las calificaciones del alumnado y la opinión sobre la docencia en dos grupos de estudiantes, unos pertenecientes a un sistema tradicional de enseñanza y otros participantes en la citada experiencia de implantación del crédito europeo.
\end{abstract}

\section{Descriptores}

Rendimiento académico; Evaluación de la docencia por el alumnado; Espacio Europeo de Educación Superior; Educación Superior.

\section{Introducción}

Los esfuerzos por armonizar los sistemas universitarios de las universidades europeas, a pesar de la creencia de parte de la comunidad universitaria, no partieron de la Declaración de Bolonia (1999), sino de la reunión de
París en la Universidad de la Sorbona en 1998, en la cual los responsables de Educación de Francia, Alemania, Italia, y Reino Unido firmaron un acuerdo que fue el origen de los procesos de convergencia. Esta reunión fue seguida de la Declaración de Bolonia en 1999, en la cual ya participaron 31 estados, matizando y clarificando los acuer- 
Molero Lopez-Barajas, D. (2007). Rendimiento académico y opinión sobre la docencia del alumnado participante en experiencias piloto de implantación del Espacio Europeo de Educación Superior.

RELIEVE, v. 13, n. 2, p. 175-190. http://www.uv.es/RELIEVE/v13n2/RELIEVEv13n2_2.htm

dos de los diferentes estados europeos en relación con la Educación Superior. Pero éstos no han sido los únicos puntos de encuentro para configurar el futuro marco europeo de educación superior, otras reuniones como la de Praga (2001), Berlín (2003), Bergen (2005) y Londres (2007), han servido para consolidar las ideas que originaron el proceso que se ha venido desarrollando en la última década.

En los últimos años, se ha producido un incremento muy importante en el número de experiencias de implantación del Sistema de Créditos Europeos dentro de la progresiva adaptación al Espacio Europeo de Educación Superior (a partir de ahora EEES) en las Universidades Españolas (Cruz de la, 2003; Gata, Bautista y Mora, 2003; Alba, 2005; Cabello y Antón, 2005, Hernández, 2005; Sánchez y Zubillaga, 2005; Pereyra-García, Sevilla y Luzón, 2006; Sánchez, 2006; Teichler, 2006). No obstante, no todos los esfuerzos han sido satisfactorios, bastantes experiencias se han abandonado por diferentes motivos. Pero inevitablemente, las universidades españolas tienen que sobreponerse a estos inconvenientes, que en muchos casos son de carácter organizativo, al ser los grupos de estudiantes muy numerosos no permitiendo el correcto funcionamiento de los procesos de enseñanza y aprendizaje.

La experiencia que presentamos tuvo su origen en el curso académico 2003/2004, cuando la Facultad de Humanidades y Ciencias de la Educación de la Universidad de Jaén coordinó la Comisión Académica de Titulación (CAT) de Maestro/a Especialidad Educación Musical de las Universidades Andaluzas. A partir de estos encuentros se elaboró un documento clave para la posterior elaboración de las Guías particulares ECTS de la Titulación en las distintas Universidades Andaluzas en el que se establecía el perfil de la titulación, salidas profesionales, competencias específicas, primera aproximación y adaptación de las asignaturas troncales de la titulación al Crédito Europeo y el consenso en el número de créditos y ubicación de las materias en los diferentes cursos del título (Díez y Ayala, 2006).

Desde el año 2004, la Junta de Andalucía y el MEC han ofertado incentivos en diferentes convocatorias para participar en la implantación de experiencias piloto del EEES. La Titulación Maestro/a Educación Musical de la Universidad de Jaén aprovechó con ilusión, aunque con alguna reticencia salvable finalmente, estas oportunidades por la posibilidad que se brindaba para la formación del profesorado en la nueva metodología (cursos de creación de páginas webs y docencia online, enseñanza y aprendizaje por competencias, proyectos de innovación docente, etc.), organización de jornadas y congresos, intercambio de experiencias con profesorado de otras universidades con experiencia en el crédito europeo, etc.

Esto originó que en el curso académico 2004/2005 se iniciara el primer nivel de la Experiencia Piloto ECTS en la titulación Maestro/a Educación Musical en nuestra Universidad, junto a las titulaciones de Maestro/a E. Educación Infantil, Psicología, Filología Inglesa, editando el servicio de publicaciones de la Universidad las Guías particulares en formato CD-Rom que fueron facilitadas al profesorado y alumnado de las mismas. En el siguiente curso, 2005/2006, se incorporaron dos nuevas titulaciones (Química e Ingeniería Técnica Industrial en tres de sus especialidades, Electricidad, Mecánica y Química Industrial) y abandonó la experiencia Maestro/a Educación Infantil debido al elevado número de estudiantes en los grupos de prácticas y de Actividades Académicas Dirigidas y no existir la posibilidad de contratar a más profesorado. Las Guías se han publicado en la página web del Secretariado de Convergencia Europea de nuestra Universidad para poder facilitar el acceso y difundir la información. La experiencia finalizó el ciclo completo de la titulación en el curso académico 2006/07, comprendiendo los tres cursos de este título, que constituye 
Molero Lopez-Barajas, D. (2007). Rendimiento académico y opinión sobre la docencia del alumnado participante en experiencias piloto de implantación del Espacio Europeo de Educación Superior.

RELIEVE, v. 13, n. 2, p. 175-190. http://www.uv.es/RELIEVE/v13n2/RELIEVEv13n2_2.htm

uno de los referentes en implantación del EEES en la titulación de Maestro/a Educación Musical en las universidades andaluzas y españolas.

Entre las principales aportaciones de diferentes estudios sobre esta temática (Lyons, 1999; Gimeno, 2004), destacan las vías utilizadas para evaluar los procesos de enseñanza y aprendizaje del alumnado universitario dentro del EEES. Las alternativas son diversas, pero cada vez toma más peso el empleo del portafolio como herramienta evaluativa, entendiéndolo como un “... proceso dinámico mediante el cual los estudiantes reúnen información sobre su trabajo y crecimiento profesional y académico, información organizada por ellos sobre la base de la reflexión, la discusión y el consenso con colegas y con el profesor-tutor" (Lyons, 1999, p.11).

Consideramos oportuno destacar algunos trabajos relacionados con el portafolio en
Educación Superior, sin ánimo de ser exhaustivos, como los de Johnston (2004) quien analiza las finalidades evaluativas de los portafolios como alternativa a la evaluación añadiendo a la función sumativa la formativa; asimismo, merecen especial mención los estudios que analizan el empleo de portafolios electrónicos (Chappell y Schermerhorn, 1999; Russell y Butcher 1999; Agra, Gewerc y Montero, 2003; Brown, 2004); y los basados en las dificultades que presentan estas herramientas de evaluación (McWethy y Gradwell, 1998; Jensen y Harris, 1999; Wright, Knight y Pomerlau, 1999); así como el trabajo de LaBoskey (2000) el cual analiza las incompatibilidades que aparecen de manera frecuente entre el portafolios y la estructura universitaria y su sistema de evaluación. Entre los trabajos realizados en diversas universidades españolas, basados en el empleo del portafolios, destacamos los de Colás, Jiménez y Villaciervos (2005), Barragán (2005), Pozo y García (2005), entre otros.

Tabla 1. Número de descriptores "Portfolios Background Materials" en la Base de datos ERIC por años.

\begin{tabular}{|c|c|}
\hline Año & Descriptor Portfolios Background Materials \\
\hline 1998 & 111 \\
\hline 1999 & 68 \\
\hline 2000 & 82 \\
\hline 2001 & 105 \\
\hline 2002 & 79 \\
\hline 2003 & 40 \\
\hline 2004 & 50 \\
\hline 2005 & 43 \\
\hline 2006 & 42 \\
\hline Total & $\mathbf{6 2 0}$ \\
\hline
\end{tabular}

Fuente: Elaboración propia

El interés por esta herramienta evaluativa no aparece de manera exclusiva en las universidades españolas. Si realizamos una búsqueda en la base de datos ERIC (Educational Resources Information Center) del término Portfolios Background Materials, aparecen 620 registros relacionados con él entre los años 1998 y 2006 (véase Tabla 1).
Pero es necesario destacar que las iniciativas de anticipación del EEES, como la que presentamos, tienen que prever mecanismos para la evaluación de los docentes universitarios. Se ha constatado la existencia de al menos 12 fuentes para la valoración de la docencia como son el rendimiento de los estudiantes, evaluación por iguales, autoevaluación, evaluación por expertos, evaluación por superiores, evaluación por exalumnos, clima 
de clase, materiales elaborados, productividad investigadora, informes de las notas y matriculados, portafolio y encuestas de opinión al alumnado; las cuales aparecen reflejadas en diferentes estudios (Nichols, 1990; Escudero, 1993; González, 1997; Mateo, 2000; Molero y Ruiz, 2005).

En la actualidad en la gran mayoría de las universidades españolas se están utilizando las encuestas de evaluación de la docencia basadas en la opinión del alumnado, pero no podemos olvidar otros modelos de evaluación que podrían ser convenientes en el futuro, como el Modelo de Evaluación del Profesorado Universitario de Tejedor (2003), en donde se propone que la información obtenida de las encuestas al alumnado debe ser complementada con otras vías como los informes del centro, informes del departamento y autoinformes de los docentes. La situación de convergencia en la que nos encontramos y las nuevas exigencias que tienen que cumplir los docentes deben originar en el futuro inmediato la adopción de nuevos modelos de evaluación por la comunidad universitaria.

Existen numerosos trabajos de revisión de investigaciones basadas en la evaluación de la docencia a través de encuestas de opinión al alumnado, como los realizados en los Estados Unidos (Feldman, 1978; Good y Mulryam, 1990; Marsh, 1984, 1987; Marsh y Roche, 1993; Wilson, 1999; Russell y Gadberry, 2000), así como en Canadá (Piccinin, 1999; Beran y Violota, 2005; Beran, Violato, Kline, Frideres, 2005), los desarrollados en Australia (Marsh y Roche, 1992; Bedggod y Pollard, 1999; y Neuman, 2000); así como los trabajos realizados en Asia (Mahmoud, 1991; Marsh, Hau, Chung y Siu, 1997; Kember y Wong, 2000; Ting, 2000; Chang, 2005).

En Europa podemos destacar los estudios de aplicación de encuestas originarias de otros países realizados en Bélgica (de Neve y Jansen, 1982) y España (Marsh, Touron y
Wheeler, 1985), entre otros; al igual que los realizados en África (Watkins y Akande, 1992). Asimismo, existen trabajos que ofrecen sus propuestas y presentan sus modelos (Centra, 1979; Murray, 1980; Aleamoni, 1981; Cohen, 1981; Overal y Marsh, 1982; Doyle, 1983; Braskamp et al, 1984; Feldman, 1984; Marsh, 1984, 1987; Tejedor, Jato y Mínguez, 1988; Escudero, 1993; Cajide, Doval y Porto, 1996; González, 1997; Wilson, 1999; Mateo, 2000; Apodaca y Grad, 2002; Muñoz, Rios y Abalde, 2002; Tejedor, 2003; Molero y Ruiz, 2005; Renaud y Murria, 2005; Richardson, 2005; Love y Scoble, 2006), tanto en el panorama nacional como internacional.

A pesar de la existencia de diferentes trabajos sobre esta temática, han sido pocas las experiencias de evaluación de la docencia por parte del alumnado en aquellas universidades de nuestro contexto que han promovido experiencias piloto de implantación del EEES. Algunos trabajos en esta línea han sido los de Fueyo (2004), el cual analiza los modelos que se utilizan y su adecuación al EEES y cómo está planteada la evaluación de centros, titulaciones y profesores en el proceso de convergencia; el trabajo de Madrid (2005) que analiza la conexión entre la formación y la evaluación de la docencia; y el trabajo de Benito y Cruz (2006) en el cual los autores analizan las nuevas claves para la docencia en el EEES.

Mención especial merece la puesta en marcha de algunas iniciativas de las administraciones educativas como el Programa DOCENTIA de la Agencia Nacional de Evaluación y Acreditación (ANECA, 2007), el cual oferta un modelo de apoyo para la evaluación de la actividad docente del profesorado universitario en colaboración con la agencias autonómicas de evaluación y las universidades, basándose en la evaluación de tres dimensiones: planificación, desarrollo y resultados; recopila información de distintas fuentes: docentes, responsables académicos y alumnado; y emplea distintos procedimientos 
Molero Lopez-Barajas, D. (2007). Rendimiento académico y opinión sobre la docencia del alumnado participante en experiencias piloto de implantación del Espacio Europeo de Educación Superior.

RELIEVE, v. 13, n. 2, p. 175-190. http://www.uv.es/RELIEVE/v13n2/RELIEVEv13n2_2.htm

de evaluación: autoinforme del docente, informe de los responsables académicos y encuesta de opinión del alumnado.

\section{Planteamiento del problema y obje- tivos}

\section{Planteamiento del problema}

El problema de investigación que nos planteamos aborda varias cuestiones, la primera de ellas es determinar si existen diferencias entre las calificaciones obtenidas por los estudiantes que siguen un sistema de evaluación tradicional y los estudiantes que participan en experiencias piloto de implantación del Espacio Europeo de Educación Superior en el marco de la asignatura Pedagogía Diferencial.

La segunda cuestión que nos planteamos en nuestra investigación, es establecer si existen diferencias en la valoración de la docencia entre los estudiantes en función del sistema de estudios que éstos empleen en el contexto de nuestra titulación, con el fin de determinar qué sistema de enseñanza, tradicional o EEES, es el más valorado por el alumnado de la materia objeto de estudio.

\section{Objetivos}

La presente investigación tiene un doble propósito, por un lado, determinar cuáles son las calificaciones que obtiene el alumnado de dos promociones diferentes de la misma titulación desarrollándose cada una de ellas bajo una organización y estructura diferente, una con un sistema tradicional de enseñanza y otra adaptada al Espacio Europeo de Educación Superior; y por otro lado, conocer cuál es la opinión del alumnado de la muestra sobre la docencia que se imparte en cada una de las promociones, con el propósito de establecer las propuestas pertinentes para conseguir una mejora en los procesos de enseñanza y aprendizaje.

Considerando el problema de investigación planteado anteriormente, los objetivos del estudio, con carácter general, son los siguientes:

- Conocer las calificaciones medias obtenidas en la materia analizada por el alumnado en dos promociones diferentes (sistema tradicional y experiencia piloto de implantación del EEES).

- Determinar la existencia de diferencias significativas, a nivel estadístico, entre las calificaciones obtenidas por el alumnado de las dos promociones estudiadas en el marco de la asignatura analizada.

- Conocer la valoración de la docencia que realiza el alumnado sobre el docente de la asignatura estudiada en las dos promociones analizadas.

- Determinar la existencia de diferencias significativas, a nivel estadístico, entre las valoraciones de la docencia realizada por el alumnado en las dos promociones estudiadas en el contexto de la materia objeto de estudio.

Entendemos, aunque no esté en nuestras manos, que los resultados obtenidos pueden ser de utilidad para la toma de decisiones de mejora dentro de los planes de innovación docente que se están realizando en nuestro contexto.

\section{Método}

\section{Muestra}

La muestra del estudio responde a un muestreo no probabilístico de tipo accidental o casual (Latorre, del Rincón y Arnal, 2003, p. 82). Los estudiantes que componen la muestra son los estudiantes de segundo curso de la titulación de Maestro/a especialidad Educación Musical de la Universidad de Jaén que cursaron la asignatura obligatoria Pedagogía Diferencial en dos promociones diferentes, la primera de ellas correspondiente al curso académicos 2004/05 y la segunda en el curso 2005/06. Los estudiantes del primer grupo (Curso académico 2004/05) desarrollaron los procesos de enseñanza y aprendizaje bajo el sistema de enseñanza tradicional "presencial”, mientras que los estudiantes 
Molero Lopez-Barajas, D. (2007). Rendimiento académico y opinión sobre la docencia del alumnado participante en experiencias piloto de implantación del Espacio Europeo de Educación Superior.

RELIEVE, v. 13, n. 2, p. 175-190. http://www.uv.es/RELIEVE/v13n2/RELIEVEv13n2_2.htm

del segundo grupo (Curso académico 2005/06) cursaron sus estudios dentro de una Experiencia Piloto de Implantación del EEES.

La distribución de la muestra aparece reflejada en la Tabla 2. El 37,5\% de los estudiantes desarrollaron sus estudios bajo un sistema de enseñanza tradicional y 62,5\%, restante, cursó estudios dentro del Plan Experimental de Implantación de Espacio Europeo de Educación Superior.

Tabla 2. Distribución de la muestra.

\begin{tabular}{|l|c|c|}
\hline & Frecuencia & $\begin{array}{c}\text { Porcentaje } \\
\text { (\%) }\end{array}$ \\
\hline Curso 2004/05 & 24 & 37,5 \\
\hline Curso 2005/06 & 40 & 62,5 \\
\hline Total & 64 & 100,0 \\
\hline
\end{tabular}

Somos conscientes de la diferencia del número de sujetos que componen cada uno de los grupos considerados, pero la distribución responde únicamente al criterio de matriculación en la materia objeto de estudio. Esta diferencia entre el número de estudiantes de cada promoción se debe probablemente al aumento de la demanda de cursar esta especialidad de reciente implantación en nuestra Universidad (recordemos que se trata de una muestra accidental). Los estudiantes de segunda matrícula o repetidores no han sido seleccionados como miembros de la muestra, ya que pretendemos estudiar al alumnado de primera matrícula de la asignatura.

\section{Instrumentos}

Los instrumentos utilizados para la recogida de información han sido diversos, en función del problema y objetivos de investigación planteados. Así, para el estudio de las calificaciones del alumnado, se han utilizado las vías de evaluación empleadas por el profesorado. En las dos promociones analizadas el docente responsable de la asignatura utilizó el mismo procedimiento para la evaluación del alumnado: un portafolios semi- estructurado (50\% valor de la asignatura) y un examen de los contenidos teóricos y prácticos (50\% calificación de la materia).

Consideramos preciso hacer una breve descripción de las características de la organización de los procesos de enseñanza y aprendizaje de los dos grupos de que comparamos, a pesar de utilizar en ambos casos las mismas técnicas para evaluar al alumnado. La organización de los procesos de enseñanza y aprendizaje del sistema tradicional está basada en la consecución y superación de unos contenidos teórico-prácticos, siendo el docente el conductor del proceso, desarrollándose las distintas actividades en torno a él. En el segundo modelo analizado, la implantación del EEES, los procesos de enseñanza y aprendizaje también diferencian contenidos teóricos y prácticos, pero aquí el alumnado es el centro del proceso, en torno a su trabajo personal y en ocasiones en grupo, siempre con la supervisión del profesorado, se consiguen los objetivos del proceso.

En relación a la valoración de la docencia por el alumnado, se utilizó como instrumento la Encuesta de opinión al alumnado sobre la actuación docente del profesorado de la Universidad de Jaén (Universidad Jaén/Centro Andaluz Prospectiva, 2006) que se viene utilizando en nuestra Universidad, está basada en una escala de valoración de 5 alternativas de respuesta (1 valoración mínima y 5 valoración máxima).

\section{Análisis y discusión de los resulta- dos}

Presentaremos los resultados diferenciándolos en función de los objetivos que nos hemos planteado, analizando, en primer lugar, todas las cuestiones relacionadas con las calificaciones de los estudiantes de la muestra, resultados académicos de los dos grupos aportando los estadísticos descriptivos obtenidos y la determinación de diferencias significativas entre las mismas. A continuación, en segundo lugar, estudiaremos las distintos 
Molero Lopez-Barajas, D. (2007). Rendimiento académico y opinión sobre la docencia del alumnado participante en experiencias piloto de implantación del Espacio Europeo de Educación Superior.

RELIEVE, v. 13, n. 2, p. 175-190. http://www.uv.es/RELIEVE/v13n2/RELIEVEv13n2_2.htm

resultados fruto de las valoraciones de la docencia realizadas por las dos cohortes consideradas, así como un estudio de las diferencias que existen en la opinión del alumnado sobre la docencia impartida por el profesor encargado de la asignatura que ha sido objeto de estudio en función del grupo al que pertenecen.

\section{Rendimiento académico de los estu- diantes}

Las calificaciones medias del alumnado aparecen reflejadas en la siguiente tabla (véase Tabla 3), en donde apreciamos que la nota media para toda la muestra de estudian- tes de las dos cohortes analizadas es de 5,9 puntos, mientras que las pertenecientes al grupo que cursó estudios bajo un sistema de enseñanza tradicional es superior (puntuación media de las calificaciones 6,11 puntos). Por último, las calificaciones medias de los participantes en la experiencia piloto de implantación del EEES, son las que reciben una menor valoración con una puntuación media de 5,77 puntos. La dispersión de las puntuaciones para el total de los encuestados y para cada uno de los grupos es similar al ser sus respectivas desviaciones típicas similares estando todas ellas en torno a una desviación estándar o típica.

Tabla 3. Calificaciones medias obtenidas por el alumnado.

\begin{tabular}{|l|c|c|}
\hline & Media & Desviación típica (Sx) \\
\hline Sistema Enseñanza Tradicional & 6,11 & 1,0957 \\
\hline Experiencia adaptación EEES & 5,77 & 1,1769 \\
\hline Total (ambos grupos) & 5,90 & 1,1494 \\
\hline
\end{tabular}

A pesar de que el rendimiento académico varía en función del tipo de organización de los procesos de enseñanza, tras la realización de un ANOVA entre las variables tipo de organización de la enseñanza y las calificaciones obtenidas (véase Tabla 4), no se han apreciado diferencias significativas a nivel estadístico al ser el valor de $F$ experimental obtenido $(F=1,316)$ menor que el valor crítico de $\mathrm{F}$ (valor crítico $F=4,00$ ), y el valor de $\mathrm{p}>0,05(p=0,256)$.

Tabla 4. ANOVA: Organización de la enseñanza y calificaciones.

\begin{tabular}{|l|c|c|c|c|c|}
\hline & $\begin{array}{c}\text { Suma de } \\
\text { cuadrados }\end{array}$ & Gl & $\begin{array}{c}\text { Media } \\
\text { cuadrática }\end{array}$ & F & Sig. \\
\hline Inter-grupos & 1,730 & 1 & 1,730 & 1,316 &, 256 \\
\hline Intra-grupos & 78,867 & 60 & 1,314 & & \\
\hline Total & 80,597 & 61 & & & \\
\hline
\end{tabular}

No hay diferencias significativas a nivel estadístico (p>0.05)

VI: Organización de la enseñanza (grupo 1: tradicional, grupos 2: EEES. VD: Calificación final de la asignatura

\section{Valoración de la docencia por el alum- nado}

En este apartado vamos a analizar la información obtenida relacionada con el segundo y tercer objetivo de la investigación. Las variables relacionadas con la valoración de la docencia (véase Tabla 5) que han sido contempladas son las que aparecen en la en- cuesta para evaluar la docencia de nuestra Universidad (UJA/CANP, 2006). La valoración global del profesorado aparece reflejada en la variable número 28 del cuestionario, la cuál hemos considerado como variable dependiente en el análisis de la varianza realizado entre las variables organización de la enseñanza (tradicional o EEES) y la valoración global de la docencia. 
Molero Lopez-Barajas, D. (2007). Rendimiento académico y opinión sobre la docencia del alumnado participante en experiencias piloto de implantación del Espacio Europeo de Educación Superior.

RELIEVE, v. 13, n. 2, p. 175-190. http://www.uv.es/RELIEVE/v13n2/RELIEVEv13n2_2.htm

Tabla 5. Variables de la encuesta de opinión al alumnado sobre la actuación docente del profesorado de la Universidad de Jaén.

\begin{tabular}{|c|c|}
\hline \multicolumn{2}{|r|}{ Variables empleadas para evaluar la actuación docente } \\
\hline 1. & El profesor informa del programa de la asignatura cuando empieza a impartirla \\
\hline 2. & Informa de los objetivos del programa de la asignatura \\
\hline 3. & El programa contiene información bibliográfica útil para el desarrollo de la asignatura \\
\hline 4. & El profesor comienza las clases a la hora fijada en el horario \\
\hline 5. & Imparte clases los días establecidos \\
\hline 6. & Cuando falta a clase da razones de su ausencia \\
\hline 7. & Cuando falta a clase lo recupera otro día \\
\hline 8. & Cuando asistes a sus tutorías en el horario establecido te atiende \\
\hline 9. & Explica los contenidos de la asignatura con seguridad \\
\hline 10. & Inicia cada tema exponiendo los objetivos del mismo \\
\hline 11. & Explica con claridad, facilitando en su caso, la toma de notas \\
\hline 12. & Destaca los aspectos fundamentales de cada tema \\
\hline 13. & Pregunta durante el desarrollo de las clases para averiguar si los alumnos tienen dificultades \\
\hline 14. & Motiva a los alumnos para que se interesen por la asignatura \\
\hline 15. & Expone ejemplos o situaciones en las que se utilizan los contenidos de la asignatura \\
\hline & En general, hace interesantes las clases \\
\hline & $\begin{array}{l}\text { Propone actividades para proponer el aprendizaje autónomo (búsqueda de información comple- } \\
\text { mentaria, trabajos, investigaciones, etc.) }\end{array}$ \\
\hline 18. & Favorece que los alumnos desarrollen una actividad reflexiva \\
\hline & $\begin{array}{l}\text { Utiliza recursos didácticos (transparencias, pizarra, medios audiovisuales, informáticos, etc.) que } \\
\text { ayudan a comprender los contenidos }\end{array}$ \\
\hline 20. & Utiliza una metodología de enseñanza adecuada a las características del grupo y de la asignatura \\
\hline & Utiliza un lenguaje claro e inteligible \\
\hline & Informa del sistema de evaluación al principio del curso \\
\hline & Utiliza diferentes procedimientos para evaluar el aprendizaje de los alumnos \\
\hline & Toma en consideración las propuestas de los alumnos sobre el desarrollo de la asignatura \\
\hline & Tiene un trato igualitario con todos los alumnos \\
\hline & Es respetuoso en el trato con los alumnos \\
\hline & Responde con interés a las intervenciones de los alumnos \\
\hline & Valora globalmente al profesor de esta asignatura \\
\hline
\end{tabular}

Fuente: Universidad de Jaén / Centro Andaluz de Prospectiva (2006)

En la Tabla 6, pueden apreciarse los estadísticos descriptivos de todas las variables sometidas a evaluación de la docencia. Hemos destacado, con un asterisco (*), las cuestiones más consideradas y las menos valoradas para toda la muestra y para cada uno de los grupos, las cuales merecen un especial comentario. Podemos observar cómo la valoración media global del docente para toda la muestra es de 4,40 puntos (re- cordemos que la valoración mínima es de 1 punto y la máxima de 5 puntos). Si diferenciamos la valoración en los dos grupos considerados, comprobamos que el profesor en el grupo del plan experimental EEES es mejor considerado (puntuación media igual a 4,50 puntos) que en el grupo de enseñanza tradicional (puntuación media igual a 4,25 puntos). 
Molero Lopez-Barajas, D. (2007). Rendimiento académico y opinión sobre la docencia del alumnado participante en experiencias piloto de implantación del Espacio Europeo de Educación Superior.

RELIEVE, v. 13, n. 2, p. 175-190. http://www.uv.es/RELIEVE/v13n2/RELIEVEv13n2_2.htm

Tabla 6. Valoración de la docencia, estadísticos descriptivos.

\begin{tabular}{|c|c|c|c|c|c|c|}
\hline & \multicolumn{2}{|c|}{$\begin{array}{c}\text { Toda la Muestra (ambos } \\
\text { grupos) }\end{array}$} & \multicolumn{2}{|c|}{$\begin{array}{c}\text { Enseñanza Tradicional } \\
\text { (curso 2004/05) }\end{array}$} & \multicolumn{2}{|c|}{$\begin{array}{c}\text { Experiencia EESS (curso } \\
2005 / 06 \text { ) }\end{array}$} \\
\hline & Media & Desv. típ. & Media & Desv. típ. & Media & Desv. típ. \\
\hline P1 & $4,7656 *$ & ,46264 & 4,8333 & ,38069 & $4,7250 *$ & ,50574 \\
\hline $\mathbf{P} 2$ & 4,4062 & ,75000 & 4,4167 & ,58359 & 4,4000 & ,84124 \\
\hline P3 & 4,5714 & ,66513 & 4,2609 & ,86431 & 4,7500 & 43853 \\
\hline P4 & 4,6719 & ,50567 & 4,5000 & ,51075 & $4,7750 *$ & ,47972 \\
\hline P5 & 4,6094 & ,74785 & 4,7083 & 69025 & 4,5500 & ,78283 \\
\hline P6 & 4,5000 & ,86307 & 4,2500 & ,91047 & 4,6667 & ,80230 \\
\hline P7 & 4,1333 & 1,28982 & $2,5000 *$ & 1,35401 & 4,6000 & ,81168 \\
\hline P8 & $4,7500^{*}$ & ,44096 & $5,0000^{*}$ & ,00000 & 4,7200 & ,45826 \\
\hline P9 & 4,7344 & ,54167 & 4,7500 & ,44233 & $4,7250 *$ & ,59861 \\
\hline P10 & 4,2222 & ,88799 & 4,3478 & ,71406 & 4,1500 & ,97534 \\
\hline P11 & 4,1562 & 83986 & 4,2500 & 60792 & $4,1000 *$ & ,95542 \\
\hline P12 & 4,4286 & ,73428 & 4,4167 & ,58359 & 4,4359 & ,82062 \\
\hline P13 & 4,3594 & ,67535 & 4,3333 & ,63702 & 4,3750 & ,70484 \\
\hline P14 & $4,0635^{*}$ & 91357 & 4,0000 & ,78019 & $4,1026 *$ & 99459 \\
\hline P15 & 4,3281 & ,69132 & 4,1667 & ,76139 & 4,4250 & 63599 \\
\hline P16 & 4,1613 & ,79328 & 4,0435 & ,63806 & 4,2308 & ,87243 \\
\hline P17 & 4,6032 & 68485 & 4,4783 & 89796 & 4,6750 & ,52563 \\
\hline P18 & 4,3281 & ,79791 & 4,3750 & ,82423 & 4,3000 & ,79097 \\
\hline P19 & $3,9375^{*}$ & 1,09653 & 2,9583* & 1,04170 & 4,5250 & 59861 \\
\hline P20 & 4,3016 & ,71018 & 4,0435 & ,76742 & 4,4500 & ,63851 \\
\hline P21 & 4,6250 & ,51946 & 4,7083 & ,46431 & 4,5750 & ,54948 \\
\hline P22 & 4,7460 & ,50699 & 4,8696* & ,34435 & 4,6750 & ,57233 \\
\hline P23 & 4,5645 & ,73821 & 4,5833 & ,65386 & 4,5526 & ,79517 \\
\hline P24 & 4,2712 & 80581 & 3,9524 & 80475 & 4,4474 & ,76042 \\
\hline P25 & 4,3750 & ,89974 & 4,3333 & ,86811 & 4,4000 & ,92819 \\
\hline P26 & 4,5313 & ,89031 & 4,4583 & ,77903 & 4,5750 & ,95776 \\
\hline P27 & 4,5079 & ,69266 & 4,5417 & ,58823 & 4,4872 & ,75644 \\
\hline P28 & 4,4062 & ,70640 & 4,2500 & ,53161 & 4,5000 & ,78446 \\
\hline
\end{tabular}

P28: Valora globalmente al docente de la asignatura

Las variables más valoradas para el total de la muestra son la número 1, El profesor informa del programa de la asignatura cuando empieza a impartirla (puntuación media igual a 4,76) y la número 8, Cuando asistes a sus tutorías en el horario establecido te atiende (puntuación media igual 4,75); y las menos valoradas las número 19, Utiliza recursos didácticos (transparencias, pizarra, medios audiovisuales, informáticos, etc.) que ayudan a comprender los contenidos y la número 14, Motiva a los alumnos para que se interesen por la asignatura, con puntuaciones medias de 3,93 y 4,06, respectivamente.

Si analizamos la opinión del grupo de enseñanza tradicional, las variables que han recibido mayor consideración son las número 8, Cuando asistes a sus tutorías en el horario establecido te atiende y la número 22, Informa del sistema de evaluación al principio del curso, recibiendo valoraciones medias de 5,0 y 4,86, respectivamente. Por el contrario, las menos valoradas son la variable número 7 
Molero Lopez-Barajas, D. (2007). Rendimiento académico y opinión sobre la docencia del alumnado participante en experiencias piloto de implantación del Espacio Europeo de Educación Superior.

RELIEVE, v. 13, n. 2, p. 175-190. http://www.uv.es/RELIEVE/v13n2/RELIEVEv13n2_2.htm

y la número 19 con puntuaciones medias de 2,5 y 2,95, respectivamente.

Finalmente, las valoraciones más destacadas del grupo participante en la experiencia piloto de adaptación al Crédito Europeo son la variable número 4, El profesor comienza las clases a la hora fijada en el horario (puntuación media igual a 4,77) y las variables número 1, El profesor informa del programa de la asignatura cuando empieza a impartirla y la número 9, Explica los contenidos de la asignatura con seguridad, ambas con una puntuación media de 4,72. En este grupo, las variables menos consideradas son la número14, Motiva a los alumnos para que se interesen por la asignatura, y la número 10, Inicia cada tema exponiendo los objetivos del mismo, con puntuaciones de 4,10 y 4,15 , respectivamente.

Tanto de manera global como en cada uno de los grupos, todas las variables han obtenido valoraciones superiores a 2,5 puntos por lo que la opinión del alumnado sobre la docencia que recibe en esta materia es satisfactoria, aunque el docente tiene varios aspectos que mejorar. Evidencias como ésta son las que le dan un carácter formativo a la evaluación del profesorado universitario permitiendo que el docente tome conciencia de sus puntos débiles y actúe en consecuencia.

Tabla 7. ANOVA: Organización de las enseñanzas y valoración docente.

\begin{tabular}{|l|c|c|c|c|c|}
\hline & $\begin{array}{c}\text { Suma de } \\
\text { cuadrados }\end{array}$ & gl & $\begin{array}{c}\text { Media } \\
\text { cuadrática }\end{array}$ & F & Sig. \\
\hline Inter-grupos &, 938 & 1 &, 938 & 1,906 &, 172 \\
\hline Intra-grupos & 30,500 & 62 &, 492 & & \\
\hline Total & 31,438 & 63 & & & \\
\hline
\end{tabular}

No hay diferencias significativas a nivel estadístico (p>0.05)

VI: Organización de la enseñanza (grupo 1: tradicional, grupos 2: EEES). VD: Valoración global del docente

Si nos centramos en la valoración global de la docencia y realizamos un análisis de la varianza (ANOVA) entre los dos grupos considerados (véase Tabla 7), apreciamos que las diferencias existentes no son significativas a nivel estadístico al obtenerse un valor de $F$ experimental $(F=1,906)$ menor que el valor crítico de $\mathrm{F}$ ( $\mathrm{F}$ valor crítico $=$ 3,92, para los grados de libertad considerados) y un valor de $\mathrm{p}>0,05(p=0,172)$. Quizá en futuros estudios con muestras más amplias obtengamos evidencias empíricas diferentes a las encontradas.

\section{Conclusiones y comentarios finales}

Como hemos comprobado en los datos presentados, no existen diferencias significativas a nivel estadístico entre las calificaciones del alumnado del sistema tradicional y las obtenidas por los participantes en experiencias de implantación del EEES. En relación con la valoración de la docencia tampoco hemos encontrado significatividad estadística en las diferencias entre los dos grupos considerados.

Será necesario realizar estudios con muestras más amplias en donde haya estudiantes de diferentes materias y distintos cursos dentro de las titulaciones implicadas en estos proyectos experimentales.

Algo está cambiando en la comunidad universitaria, se está aceptando el empleo de nuevos modelos de evaluación institucional y en concreto en lo referido a la valoración de la docencia del nuevo marco de Educación Superior. Estos modelos complementan la opinión del alumnado con evaluación por pares, informes del centro, departamentos y autoinformes. Pero es necesario pasar del interés por la temática a la puesta en marcha de los mismos, sin duda este aspecto debe ser debatido en el seno de las universidades en 
Molero Lopez-Barajas, D. (2007). Rendimiento académico y opinión sobre la docencia del alumnado participante en experiencias piloto de implantación del Espacio Europeo de Educación Superior.

RELIEVE, v. 13, n. 2, p. 175-190. http://www.uv.es/RELIEVE/v13n2/RELIEVEv13n2_2.htm

los próximos años con la intención de implementarlos a la mayor brevedad.

\section{Líneas futuras de actuación}

Para finalizar queremos destacar la necesidad de algunas líneas futuras de actuación, siendo conscientes de que el trabajo que hemos presentado no responde a un proceso acabado, todo lo contrario, debe estar abierto a propuestas de mejora que lo enriquezcan. Entre ellas podrían estar las siguientes:

- Analizar las calificaciones obtenidas por el alumnado una vez que se haya implementado de manera plena la experiencia piloto en los tres niveles de la titulación.

- Realizar futuros estudios comparativos entre diferentes materias de la misma titulación con objeto de determinar si las calificaciones académicas y la valoración de la docencia siguen las mismas tendencias del estudio que presentamos.

- Implantar nuevos modelos de evaluación de la docencia adaptados a las necesidades y demandas exigibles a los docentes del futuro EEES.

- Valorar el impacto de la enseñanza recibida por el alumnado en el Practicum de esta titulación que finaliza su aplicación en el presente curso académico y más adelante, a medio plazo, estudiar el nivel de inserción laboral de los estudiantes egresados y la relación de su empleo con los estudios cursados.

Uno de los principales retos que tenemos que superar es la disminución de la ratio docente/alumnado, para permitir el correcto funcionamiento del nuevo EEES y poder desarrollarse de la manera más adecuada los diferentes tipos de actividades en los que deben basarse los aprendizajes del alumnado. Ésto permitirá pasar de un sistema de enseñanza caracterizado por tener al docente como eje vertebrador a otro diferente en donde el epicentro sea el alumnado.

\section{Referencias bibliográficas}

Agra, M ${ }^{\mathrm{a}}$. J., Gewerc, A. y Montero, M ${ }^{\mathrm{a}}$. L. (2003). El portafolios como herramienta de análisis en experiencias de formación online y presenciales. Enseñanza, 21, 101-114. Alba, C. (Coord.) (2005). Revista de Educación, 337, monográfico de Convergencia Europea y Universidad.

Aleamoni, L. M. (1981). Student Ratings of Instruction. En J. Millman (Eds.), Handbook of Teacher Evaluation. Beverly Hills, CA: Sage.

ANECA (2007). Programa DOCENTIA, Modelo de apoyo para la evaluación de la actividad docente del profesorado universitario. Madrid: ANECA. Disponible en: http://www.aneca.es/active/docs/docentia modelo_070302.pdf[Fecha de acceso: 1211-2007].

Apodaca, P. y Grad, H. (2002). Análisis dimensional de las opiniones de los alumnos universitarios sobre sus profesores: comparación entre técnicas paramétricas y no paramétricas. Revista de Investigación Educativa, 20 (2), 385-409.

Barragán, R. (2005). El portafolio, metodología de evaluación y aprendizaje de cara al nuevo Espacio Europeo de Educación Superior. Una experiencia práctica en la Universidad de Sevilla. Revista Latinoamericana de Tecnología Educativa, 4 (1), 121-139. Disponible en: http://www.unex.es/didactica/RELATEC/su mario_4_1_htm [Fecha de acceso: 12-122006].

Bedgod, R. y Pollard, R. J. (1999). Uses and Misues of Student Opinion Surveys in Eight Australian Universities. Australian Journal of Education, 43 (2), 129-156.

Benito, A. y Cruz, A. (2006). Nuevas claves para la docencia universitaria en el Espacio Europeo de Educación Superior. Madrid: Narcea.

Beran, T. \& Violato, C. (2005). Ratings of University Teacher Instruction: How Much Do Student and Course Characteristics Really Matter? Assessment and Evaluation in Higher Education, 30 (6), 593-601. 
Beran, T., Violato, C., Kline, D. \& Frideres, J. (2005). The Utility of Student Ratings if Instruction for Students, Faculty, and Administrators: A "Consequential Validity" Study. Canadian Journal of Higher Education, 35 (2), 49-70.

Braskamp, L. A., Brandenbury, D. C. y Ory, J. C. (1984). Evaluating teaching effectiveness: A practical guide. Beverly Hills, CA: Sage Publications.

Brown, C. (2004). Design, Development and Evaluation of Electronic Portfolios for Advanced Degree Programs in Technology and Scholl Media. Chicago: Association for Educational Communications and Technology.

Cabello, J. y Antón, P. (2005). Conversaciones con el profesorado. Un estudio en cuatro universidades españolas sobre el espacio europeo y el uso de las TIC. Revista de Educación, 337, 149-167.

Cajide, J., Doval, L. y Porto, A. (1996). Perspectivas actuais da avaliación da docencia universitaria. Santiago de Compostela: Universidad de Santiago.

Centra, J. A. (1979). Determining Faculty Effectiveness. San Francisco: Jossey-Bass.

Cohen, P. A. (1981). Student ratings of instruction and student achievement: a metaanalysis of multisection validity studies. Review of Educational Research, 51(3), 281-309.

Colás, M. P., Jiménez, R. y Villaciervos, P. (2005). Portafolios y desarrollo de competencias profesionales en el marco europeo de la educación superior. Revista de Ciencias de la Educación, 204, 519-538.

Cruz de la, A. (2003). El proceso de convergencia europea: ocasión de modernizar la Universidad Española si se produce un cambio de mentalidad en gestores, profesores y estudiantes. Aula Abierta, 82, 191-216.

Chang, W. (2005). The Rewards and Challenges of Teaching Innovation in University Physics: 4 Years` Reflection. International Journal of Science Education, 27 (4), 407425.

Chappell, D. S. y Schermerhorn, J. R Jr. (1999). Using Electronic Student Portfolios in Management Education: A Stakeholder Perspective. Journal of Management Education, 23 (6), 651-662.

Díez, Ma . C. y Ayala, I. Ma . (2006). La distribución de las actividades académicas dirigidas en la franja horaria: un reto para la convergencia. En, Actas de las Jornadas de Trabajo sobre Experiencias Piloto de Implantación del Crédito Europeo en las Universidades Andaluzas. Cádiz: Universidad de Cádiz. 19-21 septiembre de 2006. Disponible en: http://www2.uca.es/orgobierno/rector/jorna das/documentos/097.pdf. [Fecha de acceso: 15-10-2006].

Doyle, K. O. (1983). Evaluating Teaching. Innovation Abstracts, 5 (27), 121-139.

Escudero, T. (1993). Enfoques modélicos en la evaluación de la enseñanza universitaria. En Actas de las III Jornadas Nacionales de Didáctica Universitaria (pp. 3-59). Las Palmas de Gran Canaria: Universidad de Gran Canaria.

Feldman, K. A. (1978). Course Characteristics and College Student's Ratings of Their Teachers: What Know and What We Don't. Research in Higher Education, 9, 199-242.

Feldman, K. A. (1984). Class size and college student's evaluations of teachers and courses: A closer look. Research in Higher Education, 21, 45-116.

Fueyo, A. (2004). Evaluación de las titulaciones, centros y profesorado en el proceso de Convergencia Europea: ¿de qué calidad y de qué evaluación hablamos?. Revista interuniversitaria de formación del profesorado, 51, 207-220.

Gata, M., Bautista, J. M. y Mora, B. (2003). La construcción del espacio europeo de Educación Superior: entre el reto y la resistencia. Aula Abierta, 82, 173-90.

Gimeno, J. (2004). El Crédito Europeo: un reto para la calidad de la enseñanza en la universidad. En UCUA, Módulo 15 de de Materiales del Proyecto de Formación del Profesorado Universitario - Guía III (520552). Córdoba: Unidad para la Calidad de las Universidades Andaluzas. 
González, J. (1997). Estudio de un instrumento para la evaluación del profesorado universitario. Tesis doctoral. Universitat de València.

Good, T. L. y Mulryan, C. (1990). Teacher ratings: A call for teacher control and selfevaluation. En J. Millman y L. DarlingHammond (Eds.), The New Handbook of Teacher Evaluation (pp. 191-215). Newbury Park, Cal: Sage Publications.

Hernández, F. (2003). Enseñar y aprender en la Universidad: una adaptación necesaria de las titulaciones al Espacio Europeo de Educación Superior. Circunstancia, Revista de Ciencias Sociales, 8, 173-190.

Jensen, K. K. y Harris, V. (1999). The Public Speaking Portfolio. Communication Education, 48 (3), 211-227.

Johnston, B. (2004). Summative Assessment of Portfolio: An Examination of Different Approaches over Outcomes. Studies in Higher Education, 29 (3), 395-412.

Kember, D. y Wong, A. (2000). Implications for Evaluations from a Study of Students' Perceptions of Good and Poor Teaching. Higher Education, 40(1), 69-97.

LaBoskey, V. K. (2000). Portfolios Here, Portfolios There ... Searching for the Essence of "Educational Portfolios". Phi Delta Kappan, 81 (8), 590-595.

Latorre, A., del Rincón, D. y Arnal, J. (2003). Bases metodológicas de la investigación educativa. Barcelona: Ediciones Experiencia.

Love, S. y Scoble, R. (2006). Developing a Quality Assurance Metric: A Panoptic View. Active Learning in Higher Education, 7(2), 129-141.

Lyons, N. (Comp.) (1999). El uso del portafolios. Propuestas para un nuevo profesionalismo docente. Buenos Aires: Amorrortu Ediciones.

Madrid, J. Ma . (2005). La formación y la evaluación docente del profesorado universitario ante el espacio europeo de educación superior. Educación Siglo XXI, 23, 49-68.

Mahmoud, M. M. (1991). Descriptive models of student decision behaviour in evaluation of higher education. Assessment and
Evaluation in Higher Education, 16 (2), 133-148.

Marsh, H. W. (1984). Student's Evaluations of University Teaching: Dimensionality, Reliability, Validity, Potential Biases, and Utility. Journal of Educational Psychology, 76 (5), 707-754.

Marsh, H. W. (1987). Student 's evaluation of university teaching: research findings. Methodological issues and directions for future research. International Journal of Educational Research, 11(3), 253-288.

Marsh, H. W. y Roche, L. A. (1992). The use of Student Evaluations of University Teaching in Different Settings: The Applicability Paradigm. Australian Journal of Education, 36 (3), 278-300.

Marsh, H. W. y Roche, L. A. (1993). The use of students' Evaluation and Individually Structured Intervention to Enhance University Teaching Effectiveness. American Educational Research Journal, 30 (1), 217-251.

Marsh, H. W., Touron, J. y Wheeler, B. (1985). Students' evaluations of university instructors: The applicability of American instruments in a Spanish setting. Teaching and teacher Education, 1 (2), 123-138.

Marsh, H.W., Hau, K., Chung, C. M. y Siu, T. (1997). Students' Evaluations of University: Chinese version of the Students' Evaluations of Educational Quality Instrument. Journal of Educational Psychology, 89(3), 568-572.

Mateo, J. (2000). La evaluación del profesorado y la gestión de la calidad de la educación. Hacia un modelo comprensivo de evaluación sistemática de la docencia. Revista de Investigación Educativa, 18(1), 7-36.

McWethy, D. y Gradwell, J. (1998). Portfolios: Viable in Vocational Education. Canadian Vocational Journal, 33 (3), 11-13.

Molero, D. y Ruiz, J. (2005). La evaluación de la docencia universitaria. Dimensiones y variables más relevantes. Revista de Investigación Educativa, 23 (1), 57-84.

Muñoz, J. M., Ríos, M. P. y Abalde, E. (2002). Evaluación docente vs. Evaluación de la calidad. RELIEVE, 8 (2), 103-134. En http://www.uv.es/RELIEVE/v8n2/RELIEV 
Molero Lopez-Barajas, D. (2007). Rendimiento académico y opinión sobre la docencia del alumnado participante en experiencias piloto de implantación del Espacio Europeo de Educación Superior.

RELIEVE, v. 13, n. 2, p. 175-190. http://www.uv.es/RELIEVE/v13n2/RELIEVEv13n2_2.htm

Ev8n2_4.htm. [Consulta realizada 15-102006].

Murray, H. G. (1980). Evaluating University Teaching: A Review of Research. Toronto: Ontario Confederation of University Faculty Association.

Neuman, R. (2000). Communicating Student Evaluation of Teaching Result: Rating Interpretation Guides. Assessment and Evaluation in Higher Education, 25(2), 121-134.

Neve, H. M. F. de y Janssen, P. J. (1982). Validity of Student Evaluation of Instruction. Higher Education, 11 (5), 543-552.

Nichols, J. O. (1990). The role of institutional research in implementing effectiveness or outcomes assessment. Association for institutional research, (37).

Overall, J. C. y Marsh, H. W. (1982). Student's evaluations of teaching: An update. American Association for Higher Education Bulletin, 35 (4), 9-13.

Pereyra-García, M. A., Sevilla, M., Luzón, A. (2006). Las universidades españolas y el proceso de la construcción del Espacio Europeo de Ecuación Superior. Limitaciones y perspectivas de cambio. Revista española de educación comparada, 12, 37-80.

Piccinin, S. (1999). How individual Consultation Affects Teaching. New Directions for Teaching and Learning, 79, 71-83.

Pozo, T. y García, B. (2005). El portafolios del alumnado: una investigación-acción en el aula universitaria. Revista de Educación, 341, 737-756.

Renaud, R. D. \& Murria, H. G. (2005). Factorial Validity of Student Ratings of Instruction. Research in Higher Education, 46 (8), 929-953.

Russell, A. y Gadberry, L. (2000). Student Opinion of Faculty: A Five Year Comparisons, Fall 1994 and spring 2000. Southwestern Oklahoma State University.

Russell, J. y Butcher, C. (1999). Using Portfolios in Educational Technology Courses.
Journal of Technology and Teacher Education, 7 (4), 279-289.

Sánchez, A. (2006). Innovación en la construcción del Espacio Europeo de Educación Superior: camino de Londres 2007. Foro de Educación, 7-8, 119-129.

Sanchez, P. y Zubillaga, A. (2005). Las universidades españolas ante el proceso de convergencia europea: Análisis de las medidas institucionales y acciones de aplicación. Revista de Educación, 337, 169-187.

Teichler, U. (2006). El Espacio Europeo de Educación Superior: visiones y realidades de un proceso deseable de convergencia. Revista española de educación comparada, 12, 113-144.

Tejedor, F. J. (2003). Un modelo de evaluación del profesorado universitario. Revista de Investigación Educativa, 21 (1), 157182.

Tejedor, F. J., Jato, E. y Mínguez, C. (1988). Evaluación del profesorado universitario por los alumnos de la Universidad de Santiago. Studia Paedagogica, 20, 73-134.

Ting, K. F. (2000). A multilevel Perspective on Students Ratings of Instruction: Lessons from the Chinese Experience. Research in Higher Education, 41(5), 637-661.

Universidad de Jaén / Centro Andaluz de Prospectiva (2006). Encuesta de opinión al alumnado sobre la actuación docente del profesorado.

Watkins, D. y Akande, A. (1992). Student Evaluations of Teaching Effectiveness: A Nigerian Investigation. Higher Education, 24 (4), 453-463.

Wilson, W. R. (1999). Students Rating Teacher. Journal of Higher Education, 70(5), 562-571.

Wright, W. A., Knight, P. y Pomerlau, N. (1999). Portfolio People: Teaching and Learning Dossiers and Innovation in Higher Education. Innovate Higher Education, 24 (2), 89-103. 
Molero Lopez-Barajas, D. (2007). Rendimiento académico y opinión sobre la docencia del alumnado participante en experiencias piloto de implantación del Espacio Europeo de Educación Superior.

RELIEVE, v. 13, n. 2, p. 175-190. http://www.uv.es/RELIEVE/v13n2/RELIEVEv13n2_2.htm

\section{ABOUT THE AUTHORS / SOBRE LOS AUTORES}

Molero López-Barajas, David (dmolero@ujaen.es). Profesor del Departamento de Pedagogía, Área de Métodos de Investigación y Diagnóstico en Educación de la Universidad de Jaén. Doctor y licenciado en Psicopedagogía, Maestro. Sus líneas de investigación se centran en el Espacio Europeo de Educación Superior, evaluación de la docencia universitaria, inserción laboral de los universitarios y formación del profesorado. Dirección postal: Universidad de Jaén, Facultad de Humanidades y CCEE. Departamento de Pedagogía Área MIDE, Campus Las Lagunillas, s/n, 23071- Jaén. Buscar otros artículos de este autor en Scholar Google

\section{Google}

\section{ARTICLE RECORD / FICHA DEL ARTÍCULO}

\begin{tabular}{|c|c|}
\hline $\begin{array}{l}\text { Reference / } \\
\text { Referencia }\end{array}$ & $\begin{array}{l}\text { Molero López Barajas, David (2007). Rendimiento académico y opinión sobre la docencia del alumnado } \\
\text { participante en experiencias piloto de implantación del Espacio Europeo de Educación Superior. } \\
\text { RELIEVE, v. 13, n. 2. http://www.uv.es/RELIEVE/v13n2/RELIEVEv13n2_2.htm. Consultado en (poner } \\
\text { fecha). }\end{array}$ \\
\hline Title / Título & $\begin{array}{l}\text { Rendimiento académico y opinión sobre la docencia del alumnado participante en experiencias piloto de } \\
\text { implantación del Espacio Europeo de Educación Superior. [Academic performance and students' teach- } \\
\text { ing assessment in pilot experiments of European Higher Education] }\end{array}$ \\
\hline $\begin{array}{l}\text { Authors / } \\
\text { Autores }\end{array}$ & Molero López Barajas, David \\
\hline $\begin{array}{l}\text { Review / } \\
\text { Revista }\end{array}$ & Revista ELectrónica de Investigación y EValuación Educativa (RELIEVE), v. 13, n. 2 \\
\hline ISSN & $1134-4032$ \\
\hline $\begin{array}{l}\text { Publication } \\
\text { date / } \\
\text { Fecha de } \\
\text { publicación }\end{array}$ & $\begin{array}{l}2007 \text { (Reception Date: } 2007 \text { January 29; Approval Date: } 2007 \text { December 21; Publication Date: } 2007 \\
\text { December 24) }\end{array}$ \\
\hline \multirow[b]{2}{*}{$\begin{array}{l}\text { Abstract / } \\
\text { Resumen }\end{array}$} & $\begin{array}{l}\text { In this work we show a study based on an analysis of academic marks as well as on a teaching valua- } \\
\text { tion of a students sample participating in a pilot experience for the European Credit System Implanta- } \\
\text { tion in the Music Teachers Instruction Course. We also detail the way of evaluating the students and the } \\
\text { resources used to valuate the teaching. We analyse the differences between the student's marks and their } \\
\text { opinion about the teaching in two students groups, one of them using a traditional teaching system and } \\
\text { the other one using such experience for the European Credit implantation. }\end{array}$ \\
\hline & $\begin{array}{l}\text { En este trabajo presentamos un estudio basado en el análisis de las calificaciones académicas y la valo- } \\
\text { ración de la docencia en una muestra de estudiantes participantes en la experiencia piloto de implanta- } \\
\text { ción del crédito europeo en la titulación de Maestro Educación Musical. Detallamos las vías empleadas } \\
\text { para evaluar al alumnado y los recursos utilizados para valorar la docencia, analizamos la existencia de } \\
\text { diferencias entre las calificaciones del alumnado y la opinión sobre la docencia en dos grupos de estu- } \\
\text { diantes, unos pertenecientes a un sistema tradicional de enseñanza y otros participantes en la citada ex- } \\
\text { periencia de implantación del crédito europeo }\end{array}$ \\
\hline Keyw & $\begin{array}{l}\text { Academic Achievement; Student Evaluation of Teacher Performance; European Higher Education Area, } \\
\text { Higher Education. }\end{array}$ \\
\hline Descriptores & $\begin{array}{l}\text { Rendimiento académico; Evaluación de la docencia por el alumnado; Espacio Europeo de Educación } \\
\text { Superior; Educación Superior. }\end{array}$ \\
\hline $\begin{array}{l}\text { Institution / } \\
\text { Institución }\end{array}$ & Universidad de Jaen (España). \\
\hline
\end{tabular}


Molero Lopez-Barajas, D. (2007). Rendimiento académico y opinión sobre la docencia del alumnado participante en experiencias piloto de implantación del Espacio Europeo de Educación Superior.

RELIEVE, v. 13, n. 2, p. 175-190. http://www.uv.es/RELIEVE/v13n2/RELIEVEv13n2_2.htm

\begin{tabular}{|l|l|}
\hline $\begin{array}{l}\text { Publication } \\
\text { site / } \\
\text { Dirección }\end{array}$ & http://www.uv.es/RELIEVE \\
\hline $\begin{array}{l}\text { Language / } \\
\text { Idioma }\end{array}$ & Spanish (Title, abstract and keywords in English ) \\
\hline
\end{tabular}

\section{Revista ELectrónica de Investigación y $\mathbf{E V}$ aluación Educativa (RELIEVE)}

\section{[ ISSN: 1134-4032 ]}

(C) Copyright, RELIEVE. Reproduction and distribution of this articles it is authorized if the content is no modified and their origin is indicated (RELIEVE Journal, volume, number and electronic address of the document).

(C) Copyright, RELIEVE. Se autoriza la reproducción y distribución de este artículo siempre que no se modifique el contenido y se indique su origen (RELIEVE, volumen, número y dirección electrónica del documento). 